\title{
A Concise Synthesis of Enantiomers of 4-Aminobutane-1,2,3-triol
}

Norma K. Dunlap*, John Drake, Andrea Ward, Tracy L. J. Salyard and Leah Martin

\author{
Middle Tennessee State University \\ Department of Chemistry \\ Murfreesboro, TN 37132 \\ ndunlap@mtsu.edu
}

\section{List of Contents}

\section{Page}

General Experimental

1

1H-NMR/13C-NMR data for (2R,4R,5R)-5-hydroxy-2-

phenyl-1,3-dioxane-4-carbaldehyde (1)

2

1H-NMR/13C-NMR data for (2R,4R,5R)-5-hydroxy-2-

phenyl-1,3-dioxane-4-carboxamide (2)

3

1H-NMR/13C-NMR data for (2R,4S,5R)-4-(aminomethyl)-2-

phenyl-1,3-dioxan-5-ol (3)

4

1H-NMR/13C-NMR data for (2R,3S)-4-aminobutane-1,2,3-triol (4) 5

1H-NMR/13C-NMR data for (2R,2'R,4S,4'S,5R,5'R)-4,4'-

azanediylbis(methylene)bis-(2-phenyl-1,3-dioxan-5-ol) (5)

6

1H-NMR/13C-NMR data for (2R,2'R,3S,3'S)-4,4'-

azanediyldibutane-1,2,3-triol) (6)

7

\section{General Experimental}

NMR spectra were obtained on a 500MHz spectrometer: 1H-NMR and 13C-NMR assignments are based on COSY, DEPT and HMQC experiments. IR spectra were obtained on an FT-IR spectrometer. Reagents and anhydrous tetrahydrofuran were purchased and used without further purification. Dichloromethane was distilled from $\mathrm{CaH}_{2}$ prior to use. 
(2R,4R,5R)-5-hydroxy-2-phenyl-1,3-dioxane-4-carbaldehyde (1).

$\underline{{ }^{1} \text { H-NMR/ } /{ }^{13} \text { C-NMR }}$
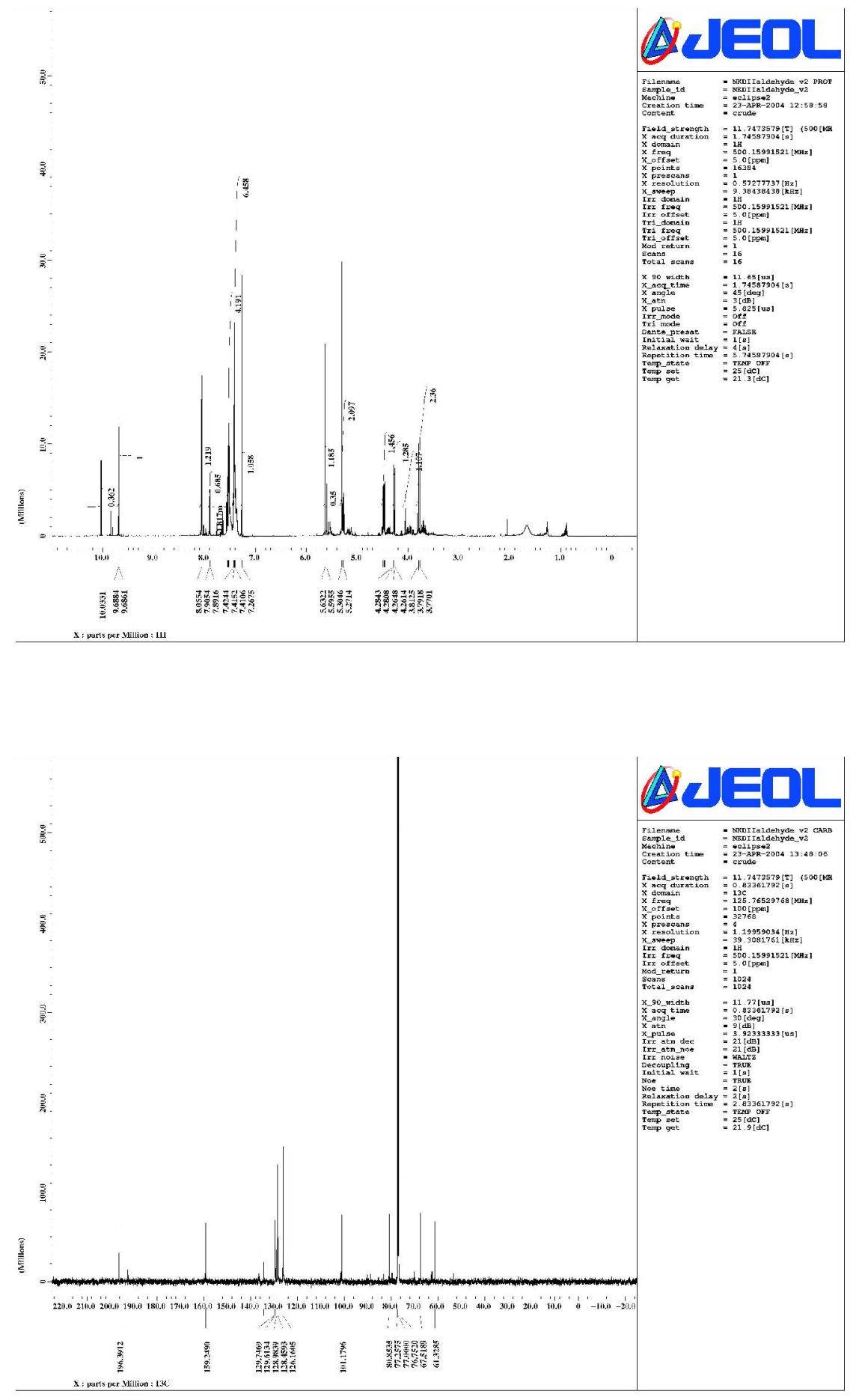
(2R,4R,5R)-5-hydroxy-2-phenyl-1,3-dioxane-4-carboxamide (2)

$\underline{{ }^{1} \mathrm{H}-\mathrm{NMR} /{ }^{13} \mathrm{C}-\mathrm{NMR}}$
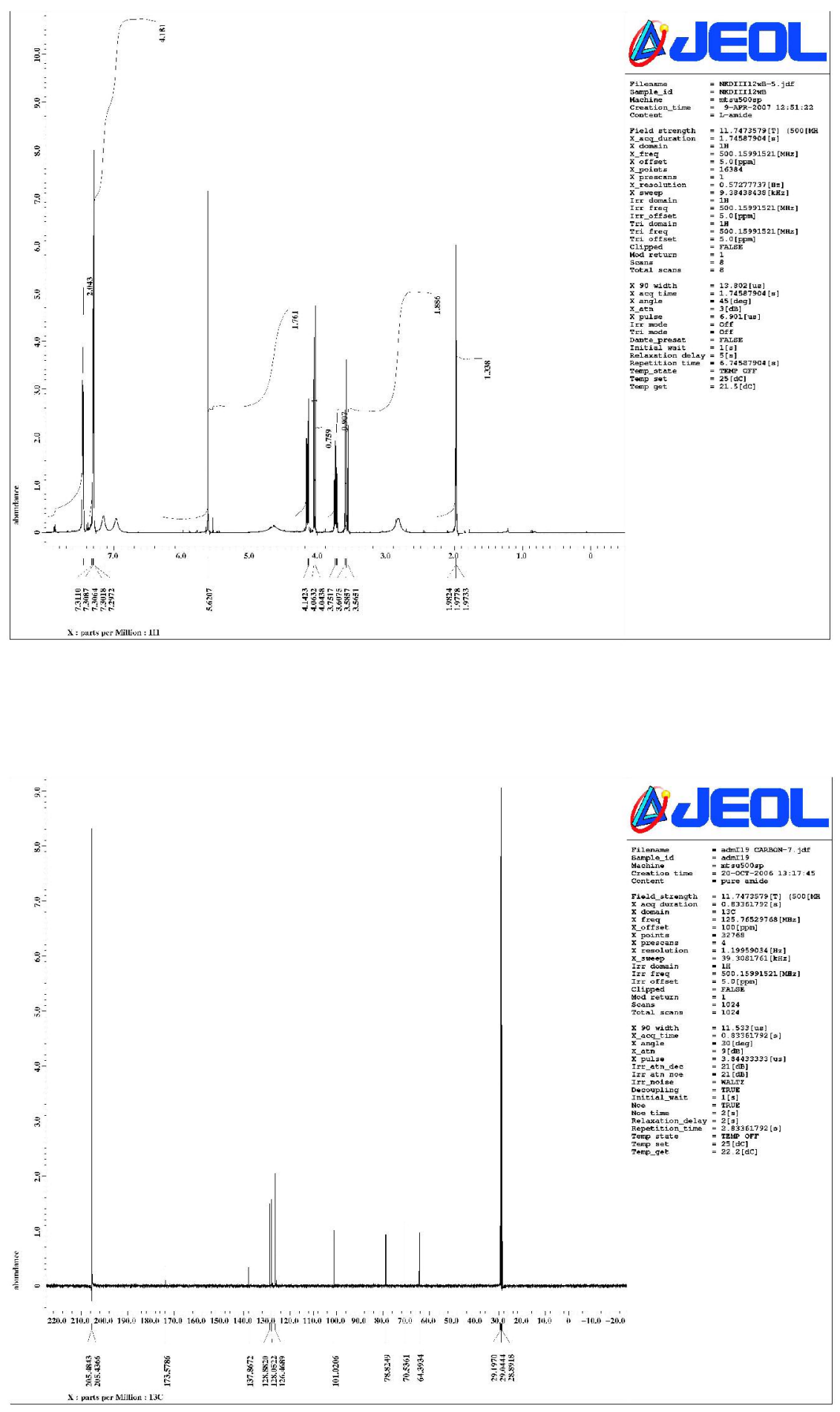
(2R,4S,5R)-4-(aminomethyl)-2-phenyl-1,3-dioxan-5-ol (3).

$\underline{{ }^{1} \mathrm{H}-\mathrm{NMR} /{ }^{13} \mathrm{C}-\mathrm{NMR}}$
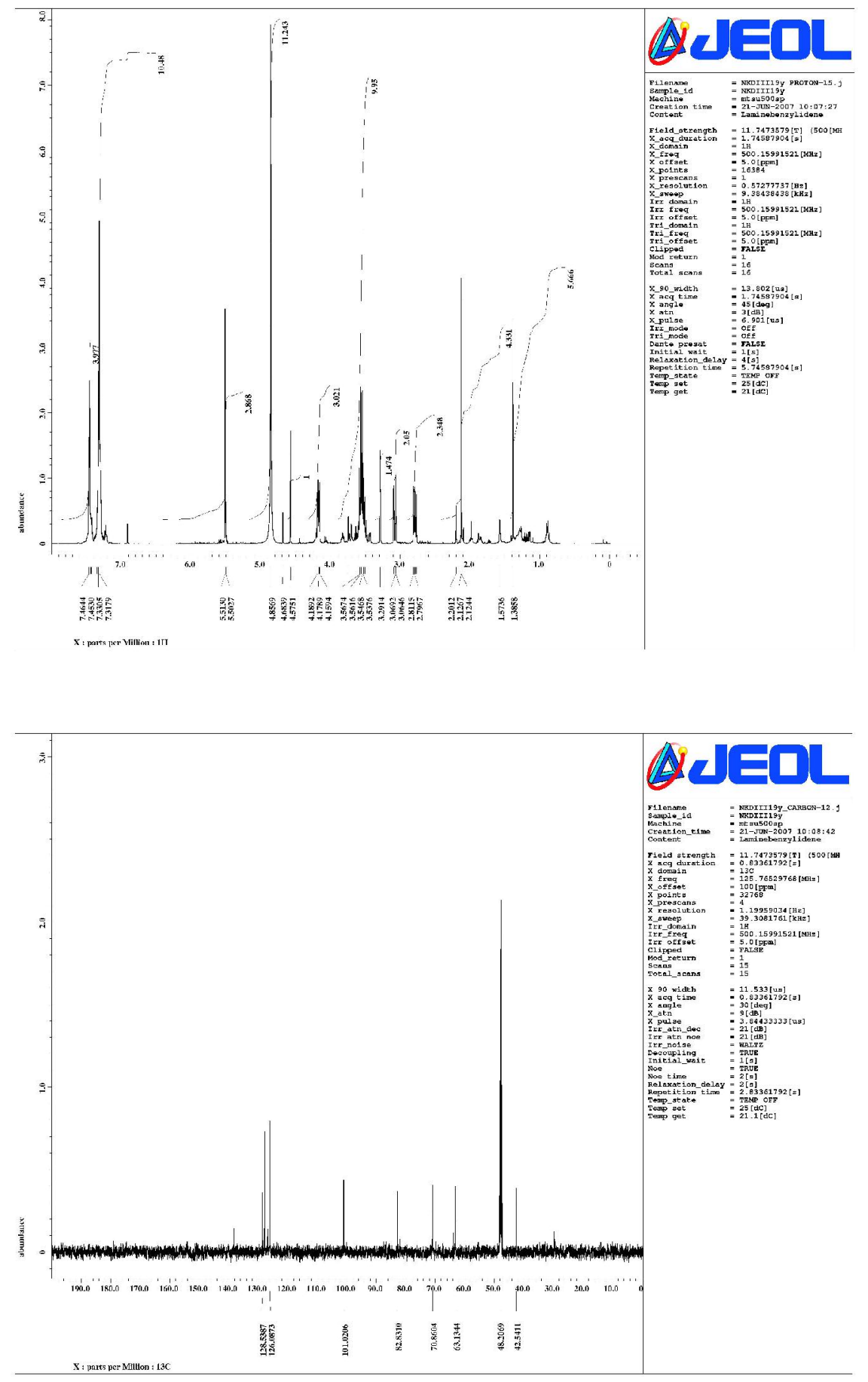
(2R,3S)-4-aminobutane-1,2,3-triol (4).

\section{${ }^{1}$ H-NMR $/{ }^{13}$ C-NMR}
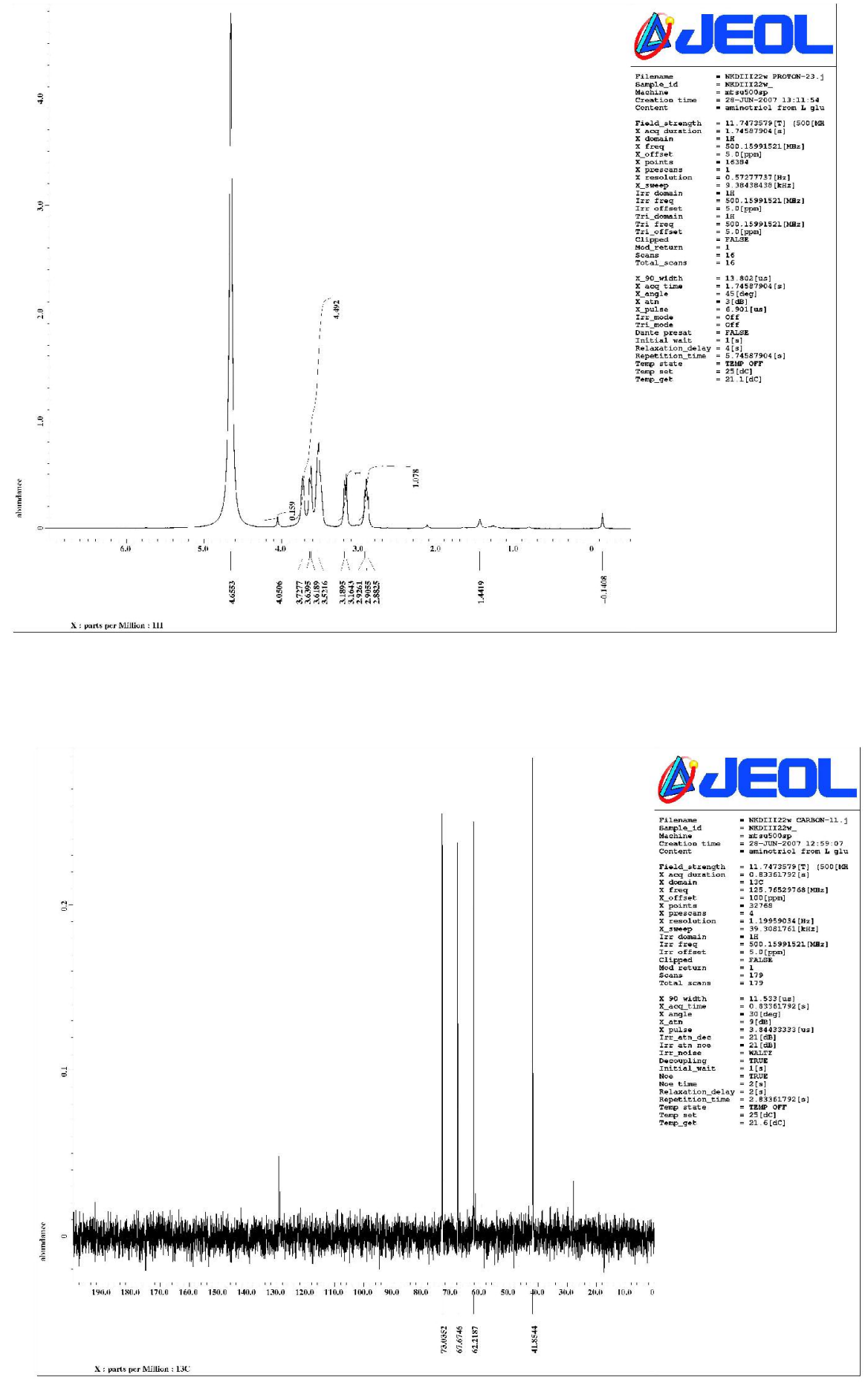
(2R,2'R,4S,4'S,5R,5'R)-4,4' -azanediylbis(methylene)bis-(2-phenyl-1,3-dioxan-5-ol) (5)

\section{$\underline{{ }^{1} \mathrm{H}-\mathrm{NMR} /{ }^{13} \mathrm{C}-\mathrm{NMR}}$}
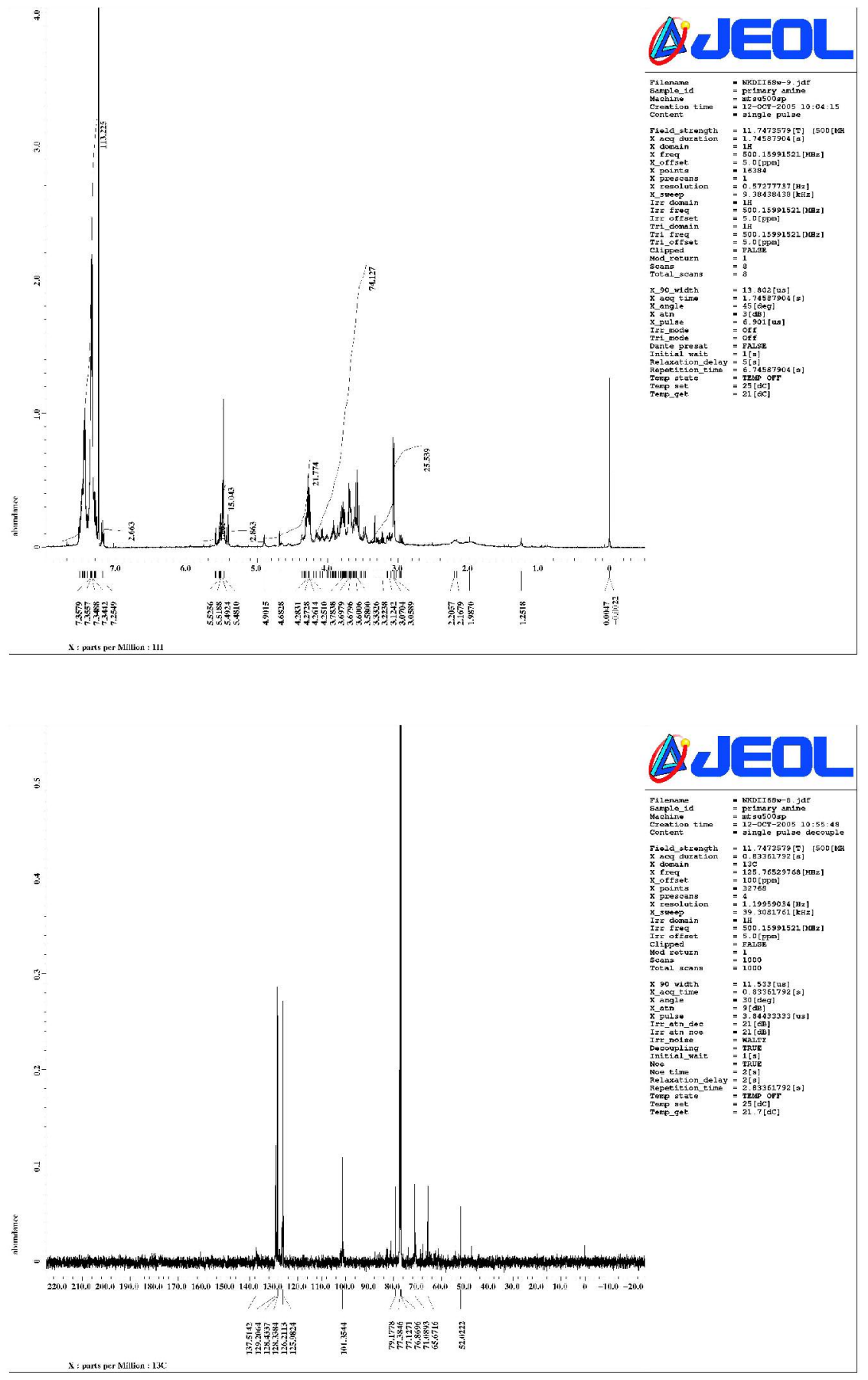
(2R,2' R,3S,3'S)-4,4' -azanediyldibutane-1,2,3-triol) (6).

\section{${ }^{1} \mathrm{H}-\mathrm{NMR} /{ }^{13} \mathrm{C}-\mathrm{NMR}$}
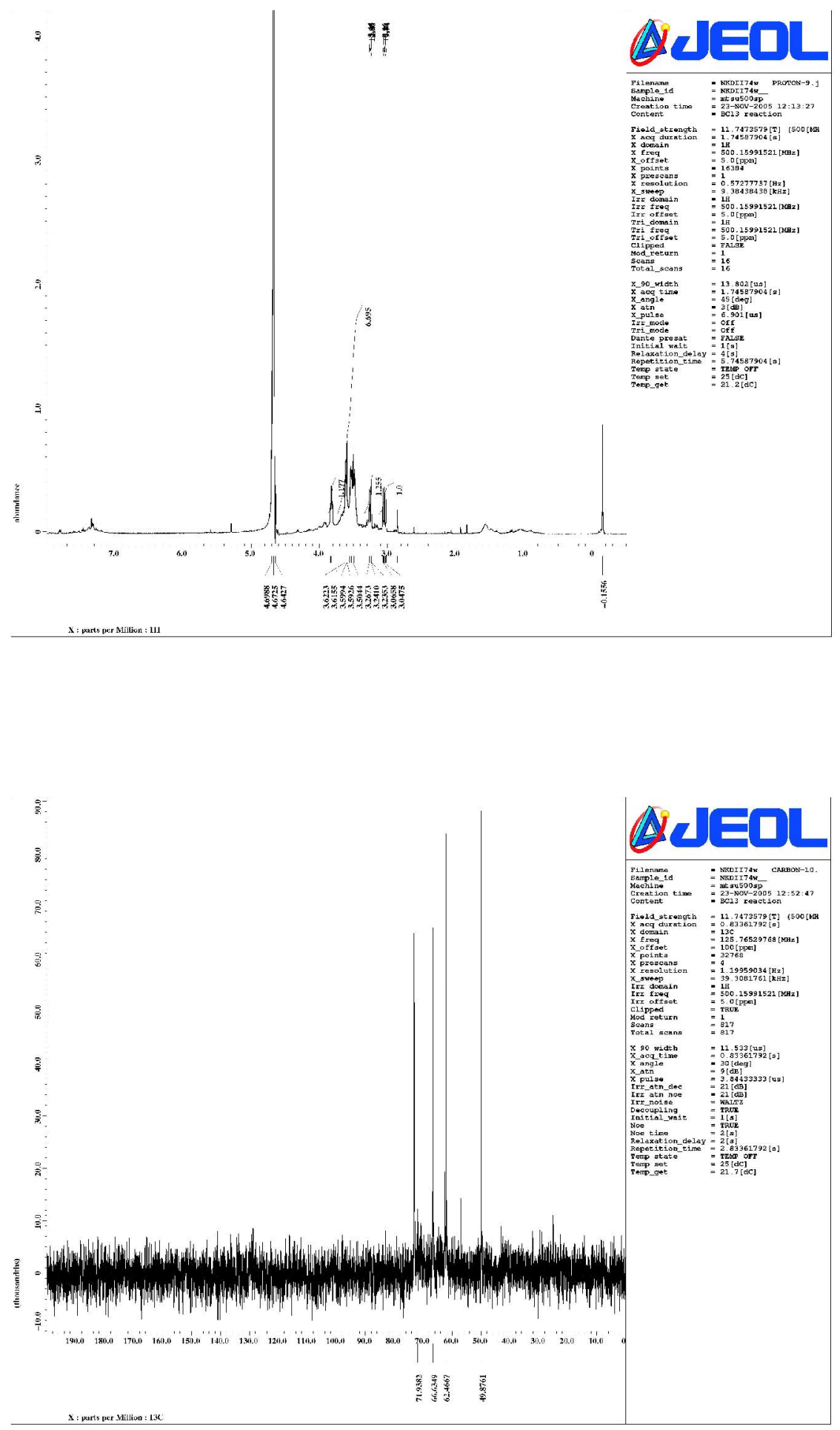